\title{
ALK-G1269A mutation in epithelioid inflammatory myofibroblastic sarcoma after progression on crizotinib: A case report
}

\author{
XIAOJING XU ${ }^{1 *}$, HONG LI $^{2 *}, \mathrm{KE} \mathrm{PENG}^{1 *}$, YIYI YU ${ }^{1}$, LINGLI CHEN $^{3}$, \\ YONG FANG $^{4}$, YIHONG SUN ${ }^{4}$, YINGYONG HOU ${ }^{3}$ and TIANSHU LIU ${ }^{1}$ \\ ${ }^{1}$ Department of Oncology, Zhongshan Hospital, Fudan University; ${ }^{2}$ Department of Oncology, \\ Jiahui International Hospital; Departments of ${ }^{3}$ Pathology and ${ }^{4}$ General Surgery, \\ Zhongshan Hospital, Fudan University, Shanghai 200032, P.R. China
}

Received December 23, 2017; Accepted November 28, 2018

DOI: $10.3892 / \mathrm{ol} .2018 .9865$

\begin{abstract}
Epithelioid inflammatory myofibroblastic sarcoma (EIMS), a specific subtype of inflammatory myofibroblastic tumors (IMT), is a relatively rare malignant mesenchymal tumor with clinical features of positive anaplastic lymphoma kinase (ALK), high invasiveness, treatment resistance and poor prognosis. Therefore, ALK inhibitors represent specific effective drugs for patients with this type of tumor. However, acquired resistance remains inevitable without a clear mechanism of action and therapeutic strategy to counteract this. Herein, a chromosomal ALK-G1269A mutation was identified using next-generation sequencing (NGS) and the mutation was confirmed by Sanger sequencing in a patient with crizotinib-resistant EIMS who benefited from treatment with the second-generation ALK inhibitor AP26113. To the best of our knowledge, a few rare cases of crizotinib-resistance in IMTs have been reported, and there are no reported cases in EIMS. In this article, we present the case of a patient with
\end{abstract}

Correspondence to: Dr Tianshu Liu, Department of Oncology, Zhongshan Hospital, Fudan University, 180 Fenglin Road, Shanghai 200032, P.R. China

E-mail: liutianshu1969@126.com

*Contributed equally

Abbreviations: EIMS, epithelioid inflammatory myofibroblastic sarcoma; ALK, anaplastic lymphoma kinase; IMT, inflammatory myofibroblastic tumor; NGS, next-generation sequencing; H\&E, hematoxylin and eosin; FFPE, formalin fixed paraffin embedded; PCR, polymerase chain reaction; IHC, immunohistochemistry; RECIST, Response Evaluation Criteria in Solid Tumors; PR, partial response; $\mathrm{PD}$, progressive disease

Key words: epithelioid inflammatory myofibroblastic sarcoma, anaplastic lymphoma kinase, kinase inhibitor, crizotinib, Brigatinib, drug resistance a secondary mutation of ALK-G1269A in EIMS, and suggest that AP26113 (Brigatinib) may represent an ideal treatment for these patients.

\section{Introduction}

Epithelioid inflammatory myofibroblastic sarcomas (EIMSs) were first described by Mariño-Enríquez in 2011 (1), and are rare malignant mesenchymal tumors commonly identified in the lung, abdominal/pelvic cavity and retroperitoneal cavity irrespective of age (2). In contrast to the conventional spindle-cell inflammatory myofibroblastic tumors (IMTs), the characteristics of EIMSs include extensive epithelial cell infiltration pathologically and a dismal prognosis (3). Given its low incidence and inadequate recognition, standard guidelines for EIMSs are presently not available. Based on experience, surgery is the primary choice for patients with localized lesions; however, in the event of recurrence or metastasis, traditional chemotherapeutic drugs such as anthracyclines are typically ineffective (2).

Similar to $50 \%$ of IMTs, EIMSs primarily express the anaplastic lymphoma kinase (ALK) protein (1). The gene encoding ALK is located on the short arm of chromosome 2 (2p23) (https://www.ncbi.nlm.nih.gov/gene/238). The protein typically manifests as RAN-binding protein 2 (RANBP2)-ALK fusion oncoprotein with distinctive nuclear membrane localization (4). Therefore, patients expressing this fusion protein may benefit from treatment with targeted ALK inhibitors such as crizotinib (5). Crizotinib is a small molecule ALK kinase inhibitor that has been approved for use in patients with various types of $\mathrm{ALK}^{+}$advanced cancer, such as non-small cell lung cancer (6). However, acquired resistance remains inevitable and largely limits the efficacy of crizotinib (7). Despite this limitation, the mechanism and therapeutic strategy for crizotinib-resistance in EIMSs have been rarely documented in the literature.

In the case presented herein article, a chromosomal ALK-G1269A mutation was identified in a patient with crizotinib-resistant EIMS by next generation sequencing (NGS) and Sanger sequencing. Treatment with the chemically 
distinct ALK inhibitor AP26113 (brigatinib) was an effective therapeutic modality in this patient, and a significant partial response was observed. The aim of the present study was to explore the resistance mechanisms and develop follow-up treatment strategies for patients with EIMSs, and to ultimately improve the symptoms and survival time of these patients.

\section{Case report}

A 28-year-old male suffering from recurrent fever, abdominal distention, night sweats and obvious fatigue since May 2016 presented at our institution in July 2018. Examination with computed tomography (CT) and magnetic resonance imaging (MRI) revealed substantial ascites and a huge caking in the abdomen and pelvis. Radical surgery was impossible due to discrete miliary nodules on the abdominal wall surface and extensive peritoneal adhesions, which were revealed by an exploratory laparotomy. Thus, a mass biopsy was performed, and the patient was diagnosed with epithelioid inflammatory myofibroblastic sarcoma composed of predominantly epithelioid cells (Fig. 1A). IHC performed as previously described (8) revealed that ALK was positive $\left(3^{+}\right)$in tumor cells (Fig. 1B), whereas other indicators, including cytokeratin, smooth muscle actin, calretinin, epithelial membrane antigen and myogenin were negative (data not shown). ALK rearrangement was assessed by fluorescence in situ hybridization (FISH) using a Vysis ALK Break Apart Probe kit (Abbott Pharmaceutical Co., Ltd., Lake Bluff, IL, USA), and tests were performed according to the manufacturer's protocol (8). ALK rearrangement was defined as positive when $>15 \%$ tumor cells exhibited split probe signals. Ten randomly chosen visual fields were observed on each section under microscope. The proportion of positive stained cells was $\sim 30 \%$ (Fig. 1C). NGS suggested that RANBP2-ALK gene fusion had occurred (Fig. 1D). Following the exploratory laparotomy, the patient's condition deteriorated rapidly, with obvious abdominal distention, dyspnea, electrolyte disturbances, a high level of uric acid $(800 \mu \mathrm{mol} / \mathrm{l})$ and extreme fatigue. From July 2016, crizotinib (200 mg) was administered to the patient twice a day orally. In September 2016, the patient's previous clinical symptoms were significantly relieved, and the response evaluation reached a partial response (PR) according to the Response Evaluation Criteria in Solid Tumors (RECIST) version 1.1 (http://recist.eortc. org/recist-1-1-2/) (Fig. 2) with slight adverse events, such as grade 1 edema and myelosuppression according to the NCI Common Terminology Criteria version 4.0 (https:// evs.nci.nih.gov/ftp1/CTCAE/). Until April 2017, the patient presented with abdominal distention, and MRI scanning confirmed the reappearance of a large amount of ascites, which was classified as progressive disease (PD). NGS, using the second aspiration biopsy specimen of the abdominal mass identified RANBP2 as fusion partner and revealed the acquisition of an ALK-G1269A mutation with a mutational abundance of $22.9 \%$ (data not shown), which was confirmed by Sanger sequencing (Fig. 3A). Primers designed were as shown in Table I. No additional mutations were detected. Treatment with AP26113 was initiated in April 2017 (90 mg daily), and the tumor-associated symptoms resolved rapidly. On July 2017, MRI scanning revealed a 50\% reduction in the abdominal mass, which was classified as a partial response (Fig. 3B). The patient is currently alive.

\section{Discussion}

Herein, we identified a chromosomal ALK-G1269A mutation in a rare and special case of a patient with EIMS who responded to treatment with AP26113, following the failure of crizotinib. Given the rarity of EIMSs, no large, well-powered clinical trials are available, with only $\sim 40$ cases being reported to date. In 2016, Yu et al (2) reviewed the clinical characteristics and pathological features of 25 cases of EIMS. The present study summarized an additional 18 cases of EIMS reported since 2016 (Table II). From these reports, certain factors, including age, sex, tumor location, tumor size, proliferation index, mitosis and necrosis degree, were not associated with clinical progression. However, positive ALK expression was closely associated with recurrence or metastasis. The positive rate of ALK rearrangements is presently unknown in EIMSs. However, according to the reported cases, several ALK fusion proteins, including RNA binding protein 2 (RANBP2)-ALK, tropomyosin 3 (TPM3)-ALK, TRK-fused Gene (TFG)-ALK and ribosome binding protein 1 (RRBP1)-ALK, were identified in EIMS (Table II). RANBP2-ALK fusion oncoproteins exhibit distinctive nuclear membrane localization, indicating that they may be sensitive to targeted kinase inhibitors, such as crizotinib (3,9-11). In the present case, the young male was diagnosed with EIMS characterized with predominant epithelioid cells and ALK positivity via immunohistochemistry. In addition, FISH using break apart probes revealed ALK rearrangement, and the NGS assay suggested that RANBP2-ALK gene fusion had occurred. These findings are consistent with the diagnostic criteria of EIMS.

Crizotinib has a significant effect in EIMS patients with ALK-rearrangement; however, it is difficult to avoid acquired resistance. In the present case report, the patient with EIMS was resistant to crizotinib after 9 months. Secondary NGS detection revealed an ALK point mutation G1269A in addition to RANBP2-ALK fusion, suggesting that the base $\mathrm{G}$ was changed to $\mathrm{C}$ at position 3806 of the ALK gene. Consequently, the coded amino acid was changed from glycine to alanine at position 1269 , which was located at the end of the adenosine triphosphate (ATP) binding pocket. The steric hindrance effect of the mutation affects ALK protein binding to oxazolidine, therefore ALK G1269A is an acquired resistance mutation in EIMS. However, only a few cases describing the mechanisms of crizotinib-resistance have been reported in IMTs and even fewer in EIMSs, as summarized in Table III (12-14).

In addition to G1269A, other secondary mutations, such as L1152R, C1156Y, F1174L, L1196M, G1202R and S1206Y, were also identified in vitro, xenograft models or patients with ALK-rearranged tumors, such as non-small-cell lung cancers (7). These patients were sensitive to high dosages of crizotinib or second-generation ALK inhibitors, such as ceritinib and alectinib $(15,16)$. In the present article, AP26113 was still effective in the patient with crizotinib resistance. AP26113 is also a second-generation ALK inhibitor with a double inhibitory effect on ALK and EGFR (17). Similar to crizotinib, 

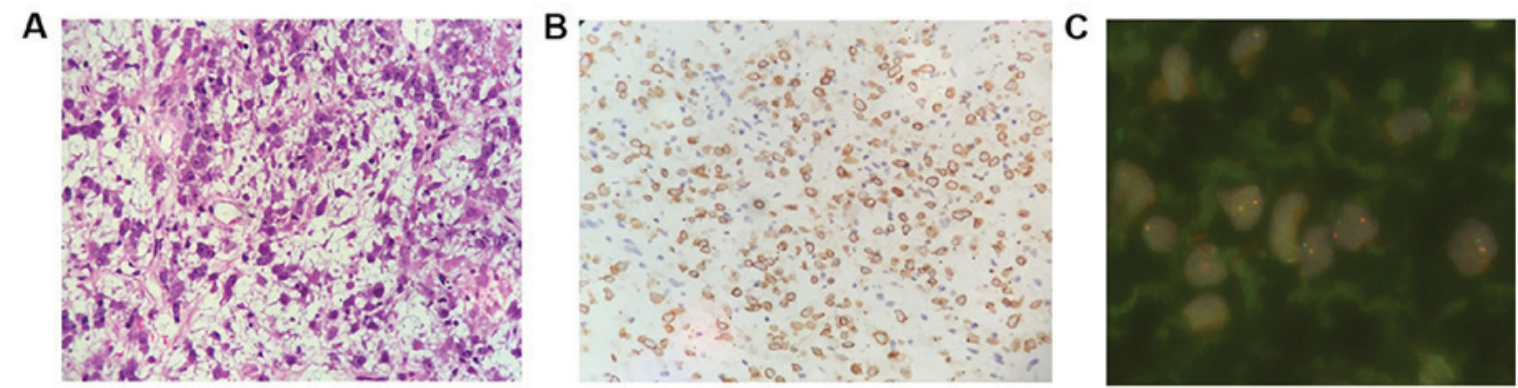

D

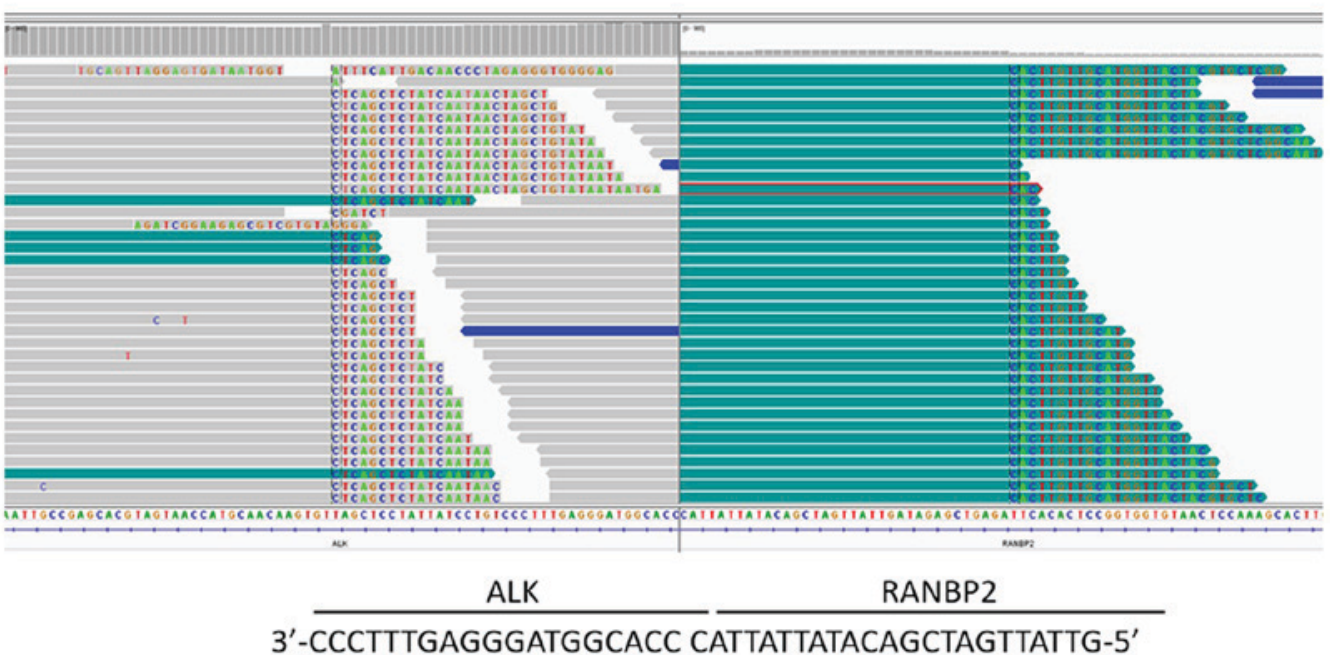

ALK

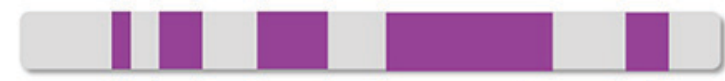

ALK/RANPB2

RANPB2

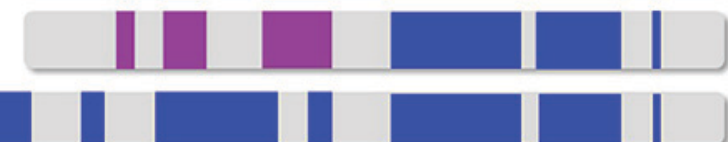

Figure 1. Characters of EIMS. (A) The tumor was predominantly composed of epithelioid cells (H\&E x100 magnification); (B) IHC revealed ALK positivity $(3+)$ in tumor cells, indicating that the tumor was ALK immunopositive (H\&E x100 magnification); (C) Fluorescence in situ hybridization using break apart probes revealed ALK rearrangement in 30\% tumor cells (H\&E x1,000 magnification); (D) NGS assays suggested RANBP2-ALK gene fusion. NGS, next generation sequencing; IHC immunohistochemistry; EIMS, epithelioid inflammatory myofibroblastic sarcoma; ALK, anaplastic lymphoma kinase.
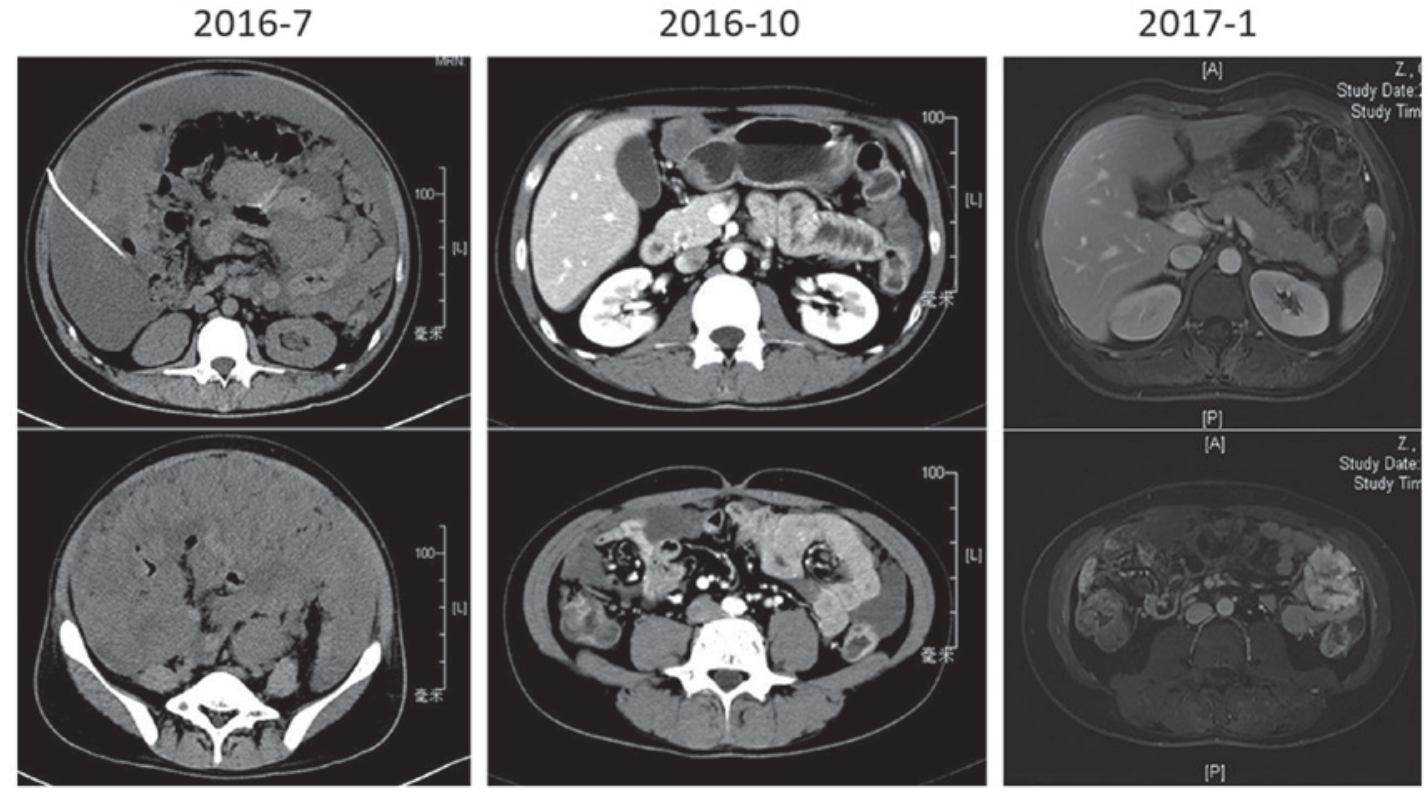

Figure 2. CT revealed a PR following treatment with crizotinib. On July 2016, computed tomography revealed massive ascites and significant caking in the abdomen and pelvis. crizotinib was administered to the patient. On October 2016, their ascites disappeared, and the response evaluation revealed a PR. Continuous PR was noted until January 2017. CT, computed tomography; PR, partial response. 
Table I. Sequences for Sanger test.

\begin{tabular}{|c|c|c|c|c|c|c|c|c|c|}
\hline Primer & Sequence $\left(5^{\prime}-3{ }^{\prime}\right)$ & $\begin{array}{c}\text { Template } \\
\text { strand }\end{array}$ & Length & Start & Stop & Temperature & GC $\%$ & $\begin{array}{c}\text { Self } \\
\text { complementarity }\end{array}$ & $\begin{array}{c}\text { Self } 3^{\prime} \\
\text { complementarity }\end{array}$ \\
\hline Forward & $\begin{array}{l}\text { TAAGGCTGTTT } \\
\text { CTCTCACAC }\end{array}$ & Plus & 20 & 213 & 232 & 55.02 & 45.00 & 3.00 & 0.00 \\
\hline Reverse & $\begin{array}{l}\text { CCATAGCCTGA } \\
\text { AAAGGAACT }\end{array}$ & Minus & 20 & 615 & 596 & 55.02 & 45.00 & 3.00 & 1.00 \\
\hline $\begin{array}{l}\text { Product length, } \\
\text { base pairs }\end{array}$ & 403 & & & & & & & & \\
\hline
\end{tabular}

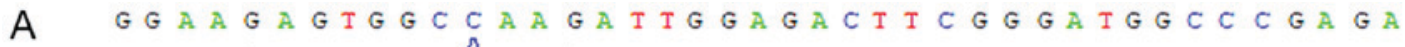

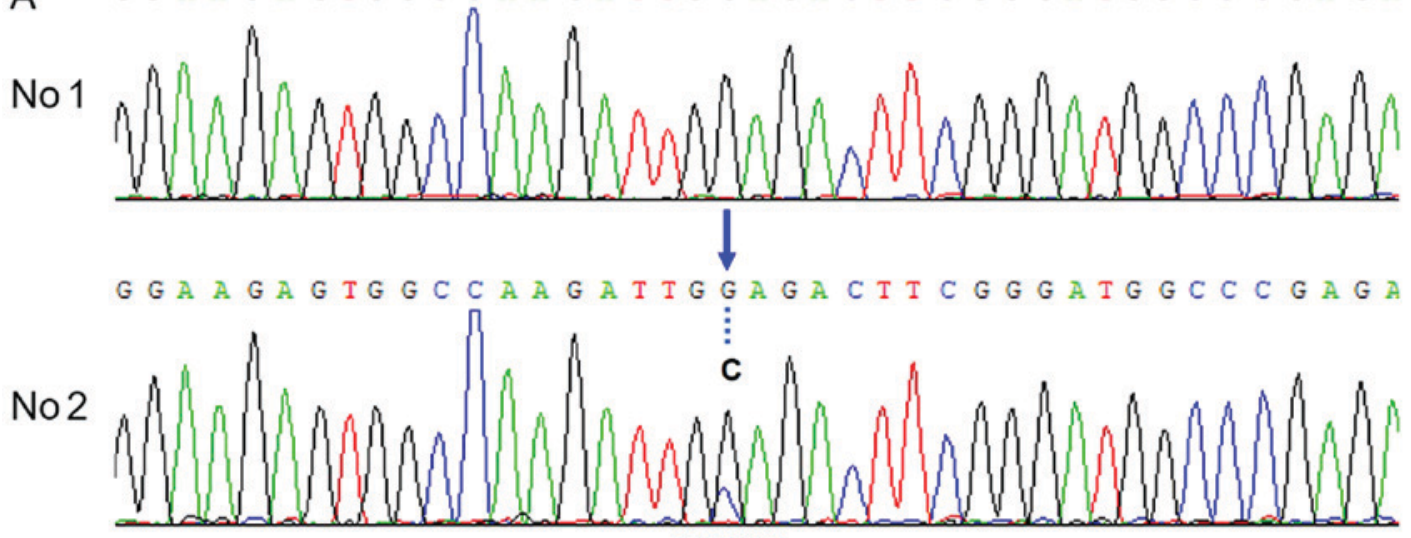

G1269A

B
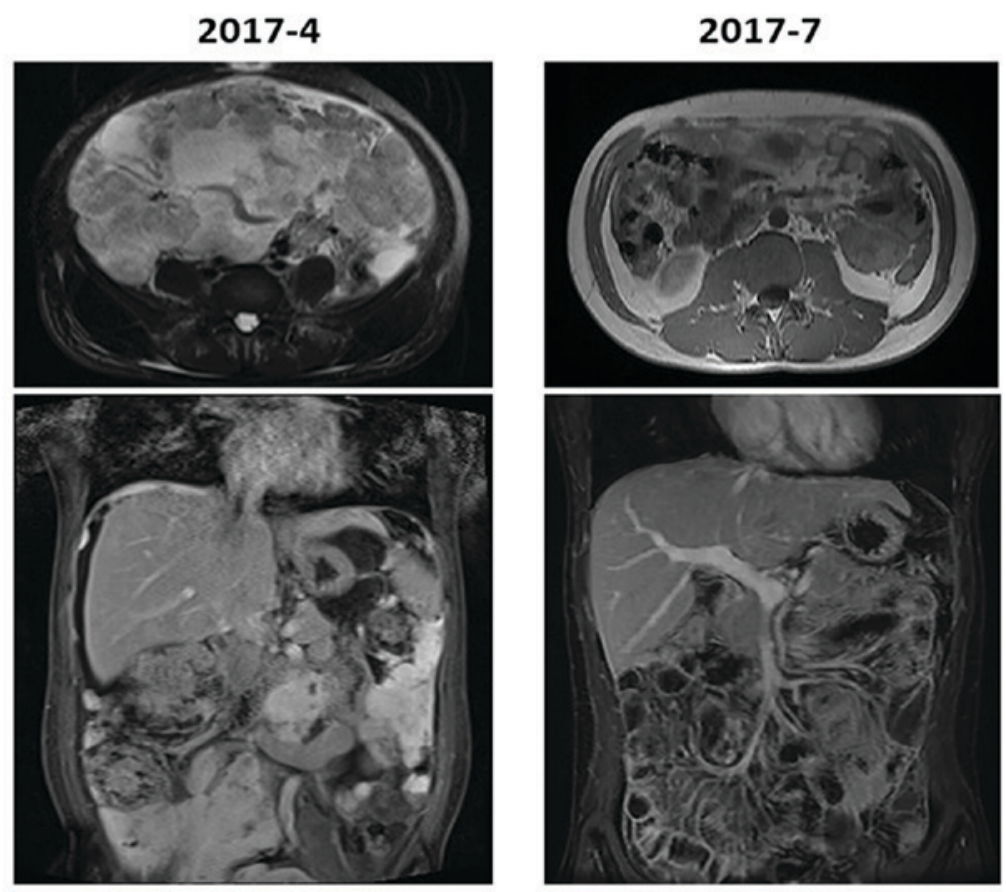

Figure 3. Assessment of resistance mutation. (A) Sanger sequencing confirmed the ALK point mutation G1269A in the crizotinib-resistant tumor tissue (No. 2). Specifically, base $\mathrm{G}$ was changed to $\mathrm{C}$ at position 3806 of the ALK gene, and the coded amino acid of was the changed from glycine to alanine. (B) On April 2017, MRI scanning confirmed the presence of a large number of ascites, this was classified as progressive disease (PD). Following the administration of AP26113, MRI scanning revealed a 50\% reduction, which was classified as a partial response again on July 2017. MRI, magnetic resonance imaging.

AP26113 could relieve the clinical symptoms of the patient, without obvious side effects. Thus, the quality of life of this patient was greatly improved. Previously, certain novel agents, such as heat shock protein (HSP) 90 inhibitors, have been demonstrated to be effective in overcoming crizotinib-resistance in preclinical models and Phase I-II studies (14). With a 


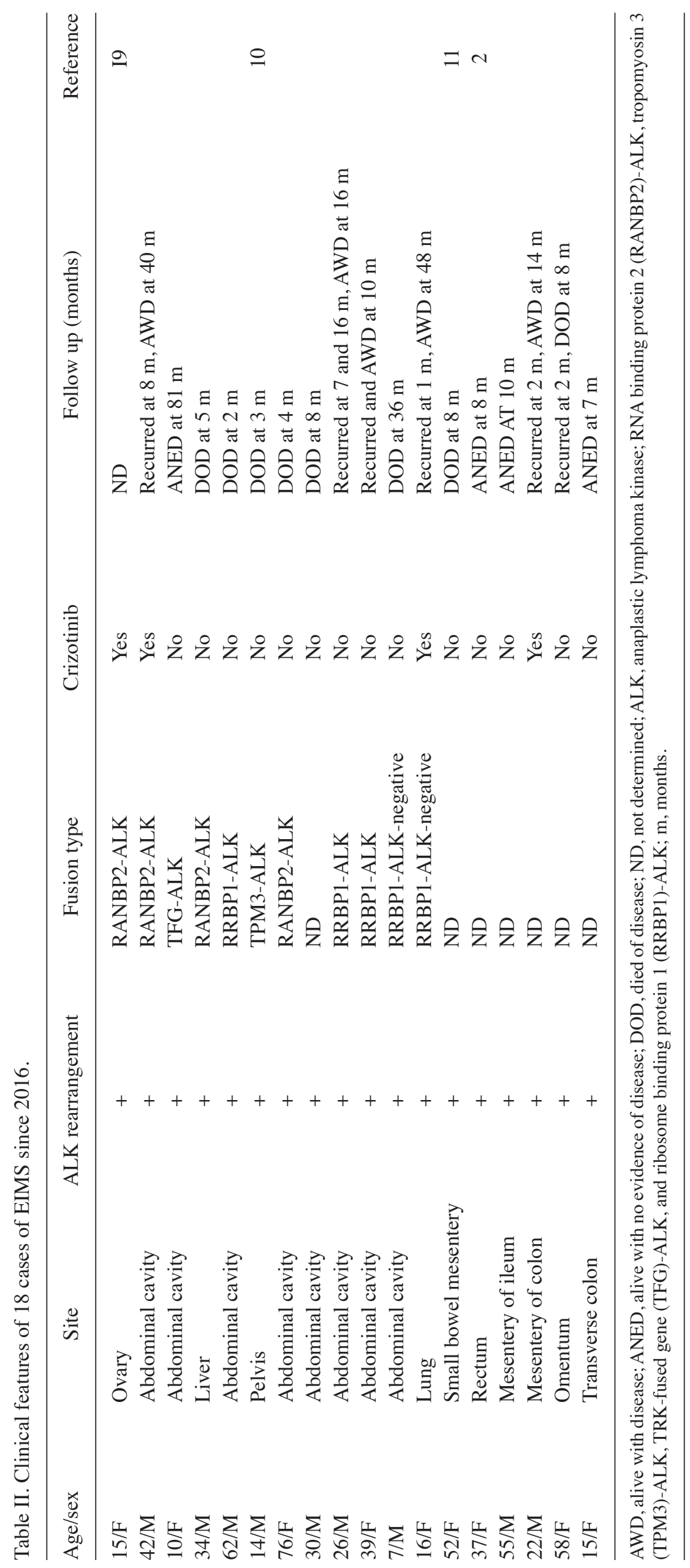


deeper understanding of EIMS, more molecular mechanisms and potential treatments will be available in the future.

\section{Acknowledgements}

The authors would like to thank Ms. Huiyan Li from the institute of Precision Medicine, 3D Medicines Inc. (Shanghai, China) for their excellent technical assistance with NGS and Sanger sequencing.

\section{Funding}

The present study was supported by the National Nature Science Foundation of China (grant no. 81502003) and the Shanghai Committee of Science and Technology (grant nos. 14ZR1406500 and 15411961900).

\section{Availability of data and materials}

The datasets used and/or analyzed during the current study are available from the corresponding author on reasonable request.

\section{Authors' contributions}

$\mathrm{XX}, \mathrm{YY}$ and TL conceived and designed the study. XX, HL and KP performed the experiments. LC and YH provided the FISH results. YF and YS performed the operation on the patient. XX wrote the paper. YY and TL reviewed and edited the manuscript. All authors read and approved the manuscript.

\section{Ethics approval and consent to participate}

The study was approved by the Ethics Committee of Zhongshan Hospital Affiliated to Fudan University (Shanghai, China). The research involved one patient and their tissues, and informed consent for use of their clinical images and tissues was obtained from the patient.

\section{Patient consent for publication}

Identifying information, including names, initials, date of birth or hospital numbers, images or statements were not included in the manuscript. And the patient has provided written informed consent for publication.

\section{Competing interests}

The authors have declared that they have no competing interests.

\section{References}

1. Mariño-Enríquez A, Wang WL, Roy A, Lopez-Terrada D, Lazar AJ, Fletcher CD, Coffin CM and Hornick JL: Epithelioid inflammatory myofibroblastic sarcoma: An aggressive intra-abdominal variant of inflammatory myofibroblastic tumor with nuclear membrane or perinuclear ALK. Am J Surg Pathol 35: 135-144, 2011.

2. Yu L, Liu J, Lao IW, Luo Z and Wang J: Epithelioid inflammatory myofibroblastic sarcoma: A clinicopathological, immunohistochemical and molecular cytogenetic analysis of five additional cases and review of the literature. Diagn Pathol 11: 67, 2016. 
3. Liu Q, Kan Y, Zhao Y, He H and Kong L: Epithelioid inflammatory myofibroblastic sarcoma treated with ALK inhibitor: A case report and review of literature. Int J Clin Exp Pathol 8: 15328-15332, 2015.

4. Kruczynski A, Delsol G, Laurent C, Brousset P and Lamant L: Anaplastic lymphoma kinase as a therapeutic target. Expert Opin Ther Targets 16: 1127-1138, 2012.

5. Kimbara S, Takeda K, Fukushima $H$, Inoue $T$, Okada $H$, Shibata Y, Katsushima U, Tsuya A, Tokunaga S, Daga H, et al: A case report of epithelioid inflammatory myofibroblastic sarcoma with RANBP2-ALK fusion gene treated with the ALK inhibitor, crizotinib. Jpn J Clin Oncol 44: 868-871, 2014.

6. Heigener DF and Reck M: Crizotinib. Recent Results Cancer Res 211: 57-65, 2018.

7. Voena $\mathrm{C}$ and Chiarle R: The battle against ALK resistance: Successes and setbacks. Expert Opin Investig Drugs 21: 1751-1754, 2012

8. Su D, Zhang D, Chen K, Lu J, Wu J, Cao X, Ying L, Jin Q, Ye Y, Xie Z, et al: High performance of targeted next generation sequencing on variance detection in clinical tumor specimens in comparison with current conventional methods. J Exp Clin Cancer Res 36: 121, 2017.

9. Fang H, Langstraat CL, Visscher DW, Folpe AL and Schoolmeester JK: Epithelioid inflammatory myofibroblastic sarcoma of the ovary with RANB2-ALK fusion: Report of a case. Int J Gynecol Pathol 37: 468-472, 2018.

10. Lee JC, Li CF, Huang HY, Zhu MJ, Mariño-Enríquez A, Lee CT, Ou WB, Hornick JL and Fletcher JA: ALK oncoproteins in atypical inflammatory myofibroblastic tumours: Novel RRBP1-ALK fusions in epithelioid inflammatory myofibroblastic sarcoma. J Pathol 241: 316-323, 2017.

11. Fang N, Yang QJ, Deng YT, Feng X, Xia HS, Zhang YG, Wang MW, Wu D, Zhou H and Guo F: Epithelioid inflammatory myofibroblastic sarcoma of small bowel mesentery: Report of a case. Zhonghua Bing Li Xue Za Zhi 46: 201-202, 2017 (In Chinese).
12. Mansfield AS, Murphy SJ, Harris FR, Robinson SI, Marks RS, Johnson SH, Smadbeck JB, Halling GC, Yi ES, Wigle D, et al: Chromoplectic TPM3-ALK rearrangement in a patient with inflammatory myofibroblastic tumor who responded to ceritinib after progression on crizotinib. Ann Oncol 27: 2111-2117, 2016.

13. Michels SYF, Scheel AH, Wündisch T, Heuckmann JM, Menon R, Puesken M, Kobe C, Pasternack H, Heydt C, Scheffler M, et al: ALKG1269A mutation as a potential mechanism of acquired resistance to crizotinib in an ALK-rearranged inflammatory myofibroblastic tumor. NPJ Precis Oncol 1: 4, 2017.

14. Sasaki T, Okuda K, Zheng W, Butrynski J, Capelletti M, Wang L, Gray NS, Wilner K, Christensen JG, Demetri G, et al: The neuroblastoma-associated F1174L ALK mutation causes resistance to an ALK kinase inhibitor in ALK-translocated cancers. Cancer Res 70: 10038-10043, 2010.

15. Nishio M, Murakami H, Horiike A, Takahashi T, Hirai F, Suenaga N, Tajima T, Tokushige K, Ishii M, Boral A, et al: Phase I study of ceritinib (ldk378) in Japanese patients with Advanced, Anaplastic lymphoma kinase-rearranged non-small-cell lung cancer or other tumors. J Thorac Oncol 10: 1058-1066, 2015.

16. Katayama R, Lovly CM and Shaw AT: Therapeutic targeting of anaplastic lymphoma kinase in lung cancer: A paradigm for precision cancer medicine. Clin Cancer Res 21: 2227-2235, 2015.

17. Huang WS, Liu S, Zou D, Thomas M, Wang Y, Zhou T, Romero J, Kohlmann A, Li F, Qi J, et al: Discovery of brigatinib (AP26113), a phosphine oxide-containing, potent, orally active inhibitor of anaplastic lymphoma kinase. J Med Chem 59: 4948-4964, 2016.

c) (i) $\odot$ This work is licensed under a Creative Commons

CY NC ND Attribution-NonCommercial-NoDerivatives 4.0 International (CC BY-NC-ND 4.0) License. 